\title{
Percepción, etimología y uso de la herpetofauna en una comunidad Embera-Dobida, en el Pacífico colombiano
}

\section{Perception, etymology and use of herpetofauna in a Embera-Dobida community in the Pacific Colombian}

\author{
Luis Eladio Rentería Moreno', Eric Yair Cuesta Ríos ${ }^{1}$, \\ Nelsy Sofía Bonilla Urrutia², Jairo Miguel Guerra Gutiérrez ${ }^{3}$
}

\begin{abstract}
Resumen
Durante octubre de 2010, caracterizamos la herpetofauna asociada con el resguardo indígena El Veintiuno, ubicado en el corregimiento de Tutunendo, Quibdó, Chocó Biogeográfico colombiano, punto caliente de diversidad global, al tiempo en que se evaluó la percepción, uso y etimología de la misma para la etnia Embera-Dobida. La información etnozoológica fue levantada mediante encuestas y diálogos interactivos con niños, jóvenes y hombres adultos (9 a 50 años), porque los hombres, al desempeñar labores en el bosque, conocen mejor la fauna. Los muestreos correspondieron a búsqueda libre realizados durante el día, por problemas de orden público. Como producto de muestreos directos en campo, se reporta 17 especies, siendo Anolis maculiventris la más abundante. El diálogo con la comunidad permitió la identificación de las especies registradas de manera directa y la adición de 15 especies más, para un total de 32; fue posible determinar que varios de los nombres asignados a las especies responden a algún atributo etológico de las mismas; además, se identificó que la principal categoría de uso de la herpetofauna fue la alimentación, enfocada en 7 especies de reptiles, siendo las tortugas el elemento más importante. La comunidad evidenció una percepción negativa sobre las serpientes las cuales son sacrificadas por prevención.
\end{abstract}

Palabras clave: Chocó, Embera, Etnozoología, Herpetofauna, Indígenas.

\section{Summary}

During October 2010, we characterize the herpetofauna associated to the resguardo El Veintiuno, located at the corregimiento of Tutunendo, Colombian Biogeographic Chocó, considered a global hot-spot of biodiversity; at the same time, the perception, use and the ethimology of herpetofauna was evaluated for the Embera-Dobida people. The etnozoological information was compiled by surveys and interactive dialogue with boys, young man, and adult males (9 to 50 years), because in the analyzed community males know better the local fauna due to their work at the forest. Free samples were implemented, during daylight hours, due to problems of public order at the community. As product of direct field samples we report 17 species of reptiles, being Anolis maculiventris the most abundant. The dialogue with members of the community, allowed the identification of all species registered in direct samples, as well as the addition of 15 more species, for a total of 32 spp; it was possible to determine that many of the assigned names to the species, respond to some ethological attribute of them; furthermore, we identified that the principle category of use of the herpetofauna was feeding, focus
1 Investigador Asociado II, Instituto de Investigaciones Ambientales del Pacífico (IIAP), Quibdó, Colombia. e-mail: erenteria@iiap.org.co ecuesta@iiap.org.co

2 Investigadora Asociada I, Instituto de Investigaciones Ambientales del Pacífico (IIAP), Quibdó, Colombia. e-mail: nbonilla@iiap.org.co

3 Asesor de Dirección, Instituto de Investigaciones Ambientales del Pacífico (IIAP), Quibdó, Colombia. e-mail: jguerra2@iiap.org.co

Recibido: 17 de octubre de 2012 Aceptado: 19 de enero de 2013 


\section{Bioetnia Volumen 10, 2013}

on seven species of reptiles, being tortoises the most important element. The community showed a negative perception on snakes, which are sacrifice in prevention.

Keywords: Chocó, Embera, Ethnozoology, Herpetofauna, Indigenous.

\section{Introducción}

En muchas regiones del Chocó, es sabido que la vida silvestre es un recurso de suma importancia para las comunidades indígenas, quienes la usan desde una perspectiva de aprovechamiento. Esta relación se percibe a través de la caza y cría de especies silvestres, siendo esta una de las actividades más importantes en la economía de subsistencia en estas culturas, la cual, combinada con otras actividades socioeconómicas como la agricultura, han permitido el establecimiento de una cultura de subsistencia (Mosquera, 2001). En relación con a lo anterior, un desafío importante es encontrar un balance entre conservación y uso, donde no se comprometa la persistencia de la fauna ni la supervivencia de la gente local. Lo cual hace que sea imperativo entender mejor cuál es el papel de la fauna en la vida de estas comunidades, porque en últimas, también nos permitiría establecer estrategias para disminuir los posibles efectos negativos del aprovechamiento del recurso.

Al respecto es importante aclarar que a pesar que la herptofauna de la región, ha sido investigada por diferentes autores generalmente estos son estudios meramente sistemáticos y ecológicos como los trabajo de Roa y Ruiz (1993), Ruiz et al. (1993, 1996), Vargas y Bolaños (1999), Grajales et al. (2003), Moreno (2000), Asprilla (2002), Rengifo et al. (2002, 2003, 2004), García y Mosquera (2005), Rentería y Rengifo (2006), Rentería et al. (2007), Moya et al. (2007) y Castro (2009), dejando de entrever el vacío de estudios desde una perspectiva de uso por parte de las comunidades indígenas, vislumbrando una ausencia de información muy importante, debido a que estas comunidades ven a muchos representantes de este grupo taxonómico como una importante fuente de proteína y a su vez, de amenaza para su integridad. Se pretende con este estudio generar información sobre la riqueza de herpetos y su relación con la comunidad indígena El Veintiuno que permita vislumbrar la forma en que esta etnia asume su biodiversidad.

Descripción del área de estudio. El resguardo indígena Embera-Dobida El Veintiuno, ubicado en corregimiento de Tutunendo, municipio de Quibdó, en el Chocó biogeográfico colombiano, identificado como punto caliente de diversidad global, se encuentra localizado a los $76^{\circ} 22^{\prime}$ de longitud oeste y $5^{\circ} 51^{\prime}$ de latitud norte; comprende una extensión de 3127 ha y está ubicado sobre la vía que de Quibdó conduce a Medellín a la altura del sitio denominado
Quebrada Carpetas. Esta área pertenece a la zona de vida Selva Pluvial Central, con una temperatura megatermal, una precipitación pluvial muy alta y una humedad relativa moderada muy húmeda (Poveda et al. 2004).

Para esta etnia la familia es la unidad social más importante dentro de la comunidad y el padre sustenta el poder familiar; la economía de subsistencia de estas familias se soporta en los productos obtenidos de sus cosechas de cultivos, así como actividades como la pesca y la obtención de especies silvestres. Este resguardo alberga 150 habitantes, 29 familias y 52 niños, que residen en viviendas (tambos) de madera, zinc y paja, donde el poblamiento consiste en que todas las familias se concentran en un solo lugar, sin embargo, cada familia tiene un terreno que es ocupado por estas y sus parcelas agrícolas, mientras que los sectores destinados para la caza, pesca y recolección de frutos, es un área comunal.

\section{Método}

Zona de muestreo. La zona de muestreo se caracterizó por estar irrigada por numerosos cuerpos de agua; además, se pudo observar parches de bosque que presentaron algún grado de intervención antrópica, por efecto de la extracción selectiva de madera; se registraron algunas parcelas agrícolas, las cuales tienen la particularidad de estar rodeadas de vegetación de desecho que se produce por el corte periódico para la limpieza de estas plantaciones; también es significativo resaltar que una importante área del bosque se caracterizó por presentar un estrato herbáceo poco desarrollado, con presencia de árboles grandes que permiten la fácil movilización y visibilidad interior.

Trabajo de campo. El trabajo de campo se realizó en el mes de octubre del 2010, tiempo en el cual se efectuaron los muestreos mediante el encuentro visual a través del método de búsqueda libre sin restricciones, en las horas del día, debido a que los problemas de orden público presentes en el área impidieron la búsqueda nocturna; los individuos se capturaron manualmente, colectándose una pequeña muestra testigo de aquellas especies que no pudieron ser satisfactoriamente identificadas en el campo. Los ejemplares colectados se sacrificaron por medio de una aplicación letal de anestésico (xilocaína), luego se inyectaron con una solución de formol al 10\%, el mismo líquido utilizado para su fijación y los individuos se etiquetaron con el número respectivo de colección para ser depositado en frascos de vidrios con alcohol etílico al $70 \%$ en la Colección Zoológica de Referencia de la Fauna Chocoana de la Universidad Tecnología del Chocó, Quibdó, Colombia. La identificación se realizó mediante la comparación con materiales de colección y con la ayuda de claves taxonómicas y guías de campo de Peter y Donoso-Barros (1970), Páez et al. (2002) 
Percepción, etimología y uso de la herpetofauna. LE Rentería Moreno et al.

y Renjifo y Lunderg (1999).

Componente etnozoológico. Este proceso se desarrolló mediante diagnóstico participativo, a través de encuestas y diálogos interactivos con la comunidad local, teniendo como objeto, niños, jóvenes y hombres adultos (9 a 50 años), porque son los hombres de la comunidad los que usualmente desempeñan labores en el bosque y conocen mejor la fauna local. Se usó la guía taxonómica de campo para la identificación de herpetos de Páez et al. (2002) y las encuestas y diálogos interactivos, estaban orientados a identificar tres puntos en particular como son: el uso que tiene los herpetos para la comunidad, la percepción de la comunidad sobre algunos grupos de herpetos y la identificación preliminar de la etimología herpetológica de la lengua Embera-Dóbida.

Sistematización y análisis de la información. Para las especies registradas en los muestreos directos, se desarrollaron listados taxonómicos de las especies; la diversidad se trabajó mediante índice directo como la riqueza de especies; se anotó la abundancia absoluta tomada como el número de individuos por especies; la información obtenida durante los diálogos interactivos fueron consignadas en el apartado de análisis etnozoológico.

\section{Resultados y discusión}

Composición taxonómica de la herpetofauna en el área de influencia de la comunidad indígena Embera-Dobida El Veintiuno. Producto de los muestreos, se registró para la zona 17 especies de herpetos, de las cuales nueve corresponden a la clase Reptilia y ocho a la clase Amphibia (Anexo 1); se registraron tres grupos de herpetos (lagartos, anuros y serpientes), siendo los lagartos el grupo más representativo (S: 8).

Las ocho especies de anfibios se distribuyeron en siete géneros y cinco familias; por otra parte los reptiles estuvieron agrupados en nueve especies, cinco géneros e igual número de familias. De la comunidad de herpeto la familia más representativa fue Polychrotidae con cinco especies, mientras que Anolis maculiventris se identificó como la especie más abundante de los muestreos (Figura 1). La representatividad de la familia Polychrotidae es atribuida a que los miembros de esta familia, se han caracterizado por experimentar un gran proceso de radiación adaptativa que les ha permitido ocupar una gran variedad de hábitat (Jackman et al., 1997). En cambio, la abundancia de $A$. maculiventris concuerda con Páez et al. (2002), quienes sostienen que esta especie puede mantener poblaciones abundantes, prefiriendo áreas abiertas (borde de bosque, senderos y áreas de cultivos), como efectivamente se evidenció durante los muestreos.

Componente etnozoológico de la comunidad indígena Embera-Dóbida El Veintiuno. Se resalta en este estudio que los diálogos con la comunidad indígena del Veintiuno permitieron la identificación no solo de las especies registradas de manera directa, sino también la adición de 15 especies de herpetos más (registros indirectos), que sumados a las 17 registradas en los muestreos, arroja un total de 32 especies para la localidad (Anexo 1).

Etimología de la comunidad indígena El Veintiuno. Con respecto a la nomenclatura que la comunidad El Veintiuno ha asignado a los herpetos, fue interesante identificar que a la especie Bothrops asper no le asignaron un nombre Embera y en cambio la reconocieron como "equis", mismo término utilizado por las comunidades afro de la región; en el caso de las lagartijas pequeñas estas son conocidas como Oiga (especialmente las especies del género Anolis); los lagartos grandes son conocidos como Gimo, las ranas son nombradas Wililikiki y Bocorrózaque. Con respecto a las serpientes dependiendo de la especie cada una tuvo un nombre en particular a excepción de las corales las cuales fueron identificadas todas como Corolachum (Micrurus spp.), otro nombre de serpiente es Jempa (Boa constrictor), este último muy similar al nombre dado por las comunidades afro donde se le conoce como Jepa; las tortugas son denominadas Pimporro (Chelydra acutirostris) y Berre-

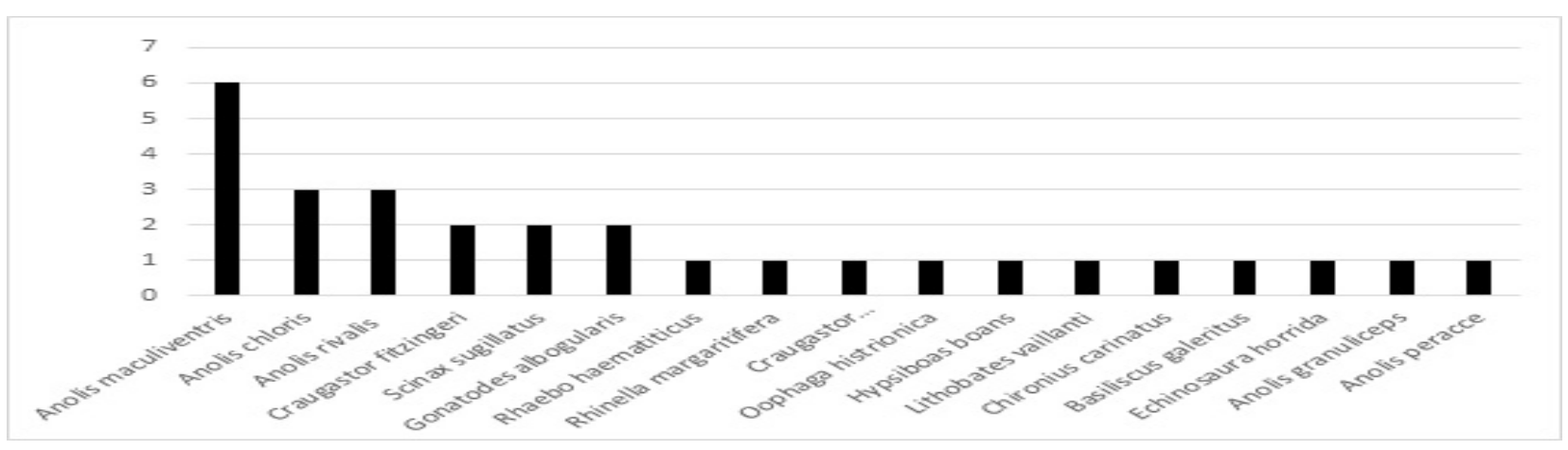

Figura 1. Abundancia de la comunidad de herpetos reportada mediante registros directos en la comunidad indígena Embera-Dobida El Veintiuno. 
Bioetnia Volumen 10, 2013

Tabla 1

Especies de herpetos usadas como fuente de proteína por la comunidad indígena Embera-Dobida El Veintiuno

\begin{tabular}{|c|c|c|c|c|}
\hline Orden & Especie & Nombre Embera & Alimentación & Control por prevención \\
\hline \multirow[t]{6}{*}{ Crocodylia } & Caiman crocodylus & Core & $x$ & \\
\hline & Basiliscos basiliscus & Opoga & $x$ & \\
\hline & Basiliscos galeritus & Opoga & $\mathrm{X}$ & \\
\hline & Boa constrictor & Jempa & & $x$ \\
\hline & Bothrops asper & Equis & & $x$ \\
\hline & Bothrops punctata & Nebe tama o & ngachun & $x$ \\
\hline \multirow[t]{6}{*}{ Squamata } & Chironius carinatus & Oconto & & $x$ \\
\hline & Iguana iguana & Opoga & $\mathrm{X}$ & \\
\hline & Lachesis muta & Birri & & $x$ \\
\hline & Leptophis ahaetulla & Dama pa & & $\mathrm{X}$ \\
\hline & Micrurus spp. & Corolachum & & $\mathrm{X}$ \\
\hline & Porthidium nasutum & lyara & & $\mathrm{X}$ \\
\hline \multirow[t]{3}{*}{ Testudinata } & Chelydra acutirostris & Pimporro & $x$ & \\
\hline & Kinosternom leucostomun & Berre berre & $x$ & \\
\hline & Rhinoclemmys nasuta & Chibigui & $\mathrm{X}$ & \\
\hline
\end{tabular}

berre (Kinosternom ssp.), las salamandras (Urodelos) en general son Moquitaparre y las cecilias (Gymnophiones) son denominadas Mevene (Anexo 1).

Explorando aún más la etimología de la nomenclatura zoológica de la comunidad indígena del Veintiuno, se halló que varios de los nombres asignados a algunas especies de herpetos, se relacionan a un atributos etológicos (comportamiento), de esta manera se identificó que el nombre Embera Cuecue de la rana Oophaga histrionica, hace referencia a la vocalización de esta especie, lo mismo sucede con Gonatodes albogularis denominado Kekedama porque según ellos cuando vocaliza en las casas canta "kekekeke", lo cual concuerda con Corl (2006), quien sostiene que los geckos son los únicos reptiles capaces de emitir sonidos vocales reales, utilizando las papilas o pliegues de la lengua para emitir una serie de "chirc" o "tic" dependiendo de la especie. Lo anterior sugiere que muy posiblemente este mismo nombre utilicen para nombrar a otros miembro del grupo de los gekos que se podrían ocurrir en la zona (Hemydactylus brokki y Tecadactylus rapicauda).

Categorías de uso de la herpetofauna de la comunidad Indígena El Veintiuno. Gracias a las encuestas y diálogo participativo se pudo identificar dos categorías de uso para la herptofauna, que son en orden de importancia alimentación y control por precaución.

Alimentación. En esta investigación se constató que el consumo de especies de herpetos en esta comunidad es muy común e importante, porque al ser Embera-Dobidad centran su área de caza principalmente a los cuerpos de aguas donde pueden encontrar diversos grupos de reptiles de gran porte proteínico. Se registraron siete especies de reptiles (las tortugas Rhinoclemmys nasuta, C. acutirostris y Kinosternon leucostomun, los lagartos Iguana iguana, Basiliscus basiliscus, B. galeritus y el cocodrilido Caiman crocodilus), utilizadas como fuente de alimento por la comunidad (Tabla 1); sin embargo los anfibios no fueron objeto de aprovechamiento por parte de los indígena Embera-Dobida El Veintiuno, lo cual se puede atribuir posiblemente al hecho de que la cantidad de biomasa de los representantes de este grupo es muy poca en comparación con los reptiles.

Control por precaución. Este fue el segundo uso dado a la herpetofauna por parte de la comunidad; sin embargo, se evidenció que es llevada a cabo exclusivamente en contra de las serpientes las cuales están representadas por al menos ocho especies (Chironius carinatus, Bothrops asper, Bothrops punctata, Lachesis muta, Porthidium nasutum, Leptophis ahaetulla, Boa constrictor y Micrurus spp.) (Tabla 1). Las serpientes en general son los únicos herpetos considerados malignos, de allí que la comunidad entrevistada afirmó que todas las serpientes son venenosas, las únicas no venenosas son la B. constrictor y L. ahaetulla y que todas las serpientes hay que matarla (a excepción ocasionalmente de la B. constrictor y L. ahaetulla), por temor, desconocimiento o como método de control para 


\section{Percepción, etimología y uso de la herpetofauna. LE Rentería Moreno et al.}

evitar accidentes. Sin embargo, es esta práctica la que más amenaza a este grupo taxonómico, debido a que la matanza indiscriminada ejercida por el ser humano es uno de los principales problemas de conservación que tradicionalmente han experimentado las serpientes (Murillo 2004).

Percepción de la comunidad indígena El Veintiuno hacia los grupos de herpetos de mayor uso. Los resultados permitieron identificar que las tortugas y las serpientes son los grupos de herpetos con que la comunidad más se relacionan. Con respecto a las tortugas, $75 \%$ de la comunidad encuestada dijo que son más avistadas en la época de verano, siendo $R$. nasuta la especie más observada en ese período, lo cual concuerda con Páez y Bock (2012) quienes sostienen que en el trópico con frecuencia las actividades reproductivas de las tortugas se concentran en verano (estación seca) época en que son más activas.

Se identificó que el método de caza de las tortugas, es la captura manual, la cual combina la habilidad del cazador con un conjunto de elementos comunes como elementos de trabajo (machete), elementos de fabricación casera (lanza o chuzo) y/o elementos del medio como garrotes o simplemente capturándolas con la mano; también mencionan una técnica de cavar las tortugas $K$. leucostomun en la que refieren así: "Nosotros salimos en verano en grupos o solos a buscar Berreberre y como ellas se encuevan, nosotros las cavamos y en un solo hueco podemos coger de 3 a 10 tortugas, pero esto solo los fines de semanas ya que los viejos dicen que allí es cuando ellas se reúnen." La anterior información es muy importante ya que confirma por primera vez para la zona lo propuesto por Rueda et al. (2007) quienes sostienen que algunos ejemplares de $K$. leucostomun pueden estivar en los bosques ribereños o enterarse entre el fango y las raíces.

Todas las personas encuestadas dijeron cazar las distintas especies de tortugas sin discriminar su talla o sexo, lo cual es un aspecto preocupante que puede estar poniendo en riesgos las poblaciones locales, porque es esta práctica la que más puede incidir en la conservación de este grupo, ya que el uso humano en muchos casos es la principal causa de la declinación de las poblaciones silvestres de tortugas y en algunos casos de su extinción (Klemens y Thorbjarnarson, 1995). Prueba de ello es que según los indígenas, la tortuga Pimporro (C. acutirostris), desde hace varios años ya no se observa en la zona debido al aprovechamiento. Lo anterior es lamentable porque los representantes de esta especie, además de ser un elemento importante para la sana dinámica trófica de los ecosistemas donde ocurren, son también una fuente importante de sustento para las comunidades indígenas por la gran cantidad de proteína que proporcionan.

Con respecto a las serpientes, se identificó que la comunidad presenta ciertas dificultades para identificar las serpientes venenosa de las no venenosas, de allí que $50 \%$ dijo no saber diferenciarlas, $25 \%$ expresó basarse en su coloración y solo el restante $25 \%$ mencionó saber diferenciarlas.

La comunidad expresa haber percibido un descenso en los encuentros ofídico (se ven mucho menos serpientes que antes), al extremo que en los alrededores de sus habitaciones ya no se observan serpientes y solo se las encuentran cuando se internan en el bosque, lo cual se puede relacionar con que $75 \%$ de la población dice sacrificar al menos una serpiente en el mes, valor muy por debajo de lo reportado por Rentería y Rivas (2009), quienes en un estudio desarrollado en la comunidad afro (Tutunendo) más próxima a la comunidad indígena El Veintiuno, los pobladores manifestaron sacrificar alrededor de 5 serpientes al mes.

La especie $B$. asper resultó ser la responsable del $50 \%$ de los encuentro ofídicos, seguida por $C$. carinatus y L. ahaetulla. El registro de B. asper como la serpiente más abundante se relaciona con que este vipérido está ampliamente distribuido en ambientes antropogenisados del sector rural donde su sobrepoblación se debe sobre todo a la disponibilidad de presas para todos los grupos de edad (mientras las áreas de cultivos aportan roedores a los individuos adultos, las charcas y los caños son sitios idóneos para la alimentación de individuos de edades neonatales y juveniles), por lo que resulta responsable de la mayoría de los accidentes ofídicos serios (Alemán 2008, Mendoza 2015). Lo anterior se ve reflejado en la psiquis de la comunidad porque $50 \%$ señala a esta especie como la serpiente a la cual le tienen mayor respecto y temor seguida por P. nasutum; sin embargo $25 \%$ de la comunidad dice no temerle a ninguna serpiente.

Finalmente aunque los encuentros ofídicos se pueden considerar relativamente escasos dentro de la zona de influencia de la comunidad indígena El Veintiuno, sus pobladores afirmaron que se han presentados accidentes ofídicos, y que tratan las mordeduras de serpientes a través del uso de plantas tradicionales de la región, con las que se lava la herida, se hacen emplastos (cubrir la herida con plantas) y finalmente se da una toma (que es un maceramiento de las mismas plantas), en cuyo caso si las lesiones son persistente y existen los medios, el enfermo es traslado a la capital de Quibdó para ser tratado en un hospital.

\section{Conclusiones}

Se registró un total de 32 especies de herpetos, los cuales evidenciaron el importante conocimiento que la comunidad Embera-Dobida El Veintiuno tiene de este grupo taxonómico al reconocer más del $80 \%$ de las especies aquí reportadas y al contribuir con el registro indirecto de 15 de ellas.

Con respecto a la información entnozoológica, se pudo 


\section{Bioetnia Volumen 10, 2013}

determinar que la relación de la comunidad con la herpetofauna, es principalmente de carácter extractivo, donde la caza de subsistencia está basada en 7 especies de reptiles, sin embargo, también hay un componente cultural importante al incluir todas las especies de serpientes en la categoría de control por prevención basados en sus mitos y creencias.

Con respecto a la etimología herpetológica de la comunidad indígena El Veintiuno, se identificó que alguno de los nombres establecidos a algunos elementos de la herpetofauna, se relacionan con un atributo etológico (comportamiento) de la misma.

Finalmente, se pudo identificar que las tortugas y las serpientes son muy posiblemente los grupos de herpetos que más se relación con esta comunidad, al ser una fuente importante de proteína y al hacer parte del misticismo y cosmovisión de la misma.

\section{Agradecimientos}

Nelsy Sofía, Jairo Miguel, Eric Yair, comunidad indígena de El Veintiuno.

\section{Literatura citada}

Alemán J. 2008. Caracterización de reptiles y percepción local hacia las serpientes en fincas ganaderas de la subcuenca del río Copán, Honduras. Tesis de maestría. Turrialba: Escuela de Posgrado del CATIE, Programa de Educación para el Desarrollo y la Conservación del Centro Agronómico Tropical de Investigación y Enseñanza; 125 pp.

Asprilla J.2002. Ecología y estructura de las comunidades de anuros presentes en dos zonas de bosque pluvial tropical (BP-T) en el departamento del Chocó (Colombia). [Tesis de grado]. Quibdó: Universidad Tecnológica del Chocó, Programa Biología con Énfasis en Recursos Naturales.

Castro F. 2009. Región natural chocoana, una de las diversidades importantes en la herpetología colombiana. En: Tailor J, Ramos Y (ed.). Memoria del Primer Congreso Regional de Herpetología. Quibdó: Universidad Tecnológica del Chocó; 48 pp.

Corl J. 2006. Gekko gecko: Tokay gecko. [En línea] University of Michigan Museum of Zoology. Animal diversity web. [Consultado: agosto 16 del 2006]. Disponible en: http://animaldiversity.ummz.umich.edu/site/ accounts/information/Gekko gecko.htm

García U, Mosquera F. 2005. Caracterización taxonómica de la comunidad de Lagartos (Squamata-Lacertilia) en el sotobosque de la Cuenca del río Cabi, Chocó. [Tesis de grado]. Quibdó: Universidad Tecnológica del Chocó "Diego Luis Córdoba", Facultad de Ciencias; 60 pp.

Grajales D, Palacios H, Echeverry CL, Gil YN. 2003. Composición y estructura de una comunidad de anuros en áreas con diferentes grados de intervención antrópica en Salero, Unión Panamericana. Capítulo IV. pp 91-102. En: Garcia F, Ramos Y, Palacios J, Arroyo J, Mena A, González M. Salero, diversidad de un bosque pluvial tropical (bp-T). Chocó: Universidad Tecnológica del Chocó e Instituto de Investigaciones Ambientales del Pacifico.

Jackman T, Losos JB, Larson A, de Queiroz K. 1997. Phylogenetic studies of convergent adaptive radiations in Caribbean Anolis lizards. In: Givnish T, Systma K (eds.). Molecular evolution and adaptiveradiation. Cambridge: Cambridge University Press; pp. 535-57.

Klemens MW, Thorbjarnarson JB. 1995. Reptiles as a food resource. Biodivers Conserv. 4: 281-98.

Mendoza J. 2015. El reto de la conservación de serpientes en Colombia. Revista Hipótesis Apuntes. 19: 38-47.

Moreno PR 2000. Preferencia de micro hábitat de una comunidad de anuros de un bosque muy húmedo tropical del corregimiento de Samurindo, municipio del Atrato, Chocó, Colombina. [Tesis de grado]. Quibdó:
Universidad Tecnológica del Chocó "Diego Luis Córdoba", Programa de Biología con énfasis en Recursos Naturales.

Mosquera J. 2001. Caracterización de la fauna de cacería (aves, mamiferos, reptiles y peces) en la comunidad de Buchado, Atrato, Medio Antioquia. [Tesis de grado]. Quibdó: Universidad Tecnológica del Chocó "Diego Luis Córdoba"; 106 pp.

Moya J, Riva T, Rentería L. 2007. Fauna anura presente en la cabecera municipal de Quibdó, Chocó, Colombia. Revista de la Asociación Colombiana de Ciencias Biológicas. 19 (Supl 1): 188.

Murillo F, Moreno E, Roa Y, Mena Y, Rengifo J. 2004. Caracterización ecológica de la ofidiofauna en el corregimiento de Pacurita, Chocó, Colombia. Revista Institucional Universidad Tecnológica del Chocó. 19: 45-9.

Páez V, Bock B. 2012. Ecología de anidación de las tortugas continentales de Colombia. Capítulo 11. Pp. 147-158. En: Páez, VP, Morales-Betancourt MA, Lasso CA, Castaño-Mora OV, Bock BC (eds.). Biología y conservación de las tortugas continentales de Colombia. Serie Editorial Recursos Hidrobiológicos y Pesqueros Continentales de Colombia. Bogotá: Instituto de Investigación de Recursos Biológicos Alexander von Humboldt (IAvH).

Páez V, Bock CB, Estrada JJ, Ortega AM, Gutiérrez PD. 2002. Guía de campo de algunas especies de anfibios y reptiles de Antioquia. Medellín: Colciencias, Universidad de Antioquia, Universidad Nacional; 136 pp.

Peter J, Donoso-Barros R. 1970. Catálogo of the Neotropical Squamata. Part II. Lizard and Amphisbaenians. Washington, DC: Smithsonian Institution Press.

Poveda C, Rojas C, Rudas A, Rangel-Ch O. 2004. Climas del Chocó Biogeográfico de Colombia. Pp. 39-89. En: Rangel J (ed.). Colombia Diversidad Biótica IV. Chocó Biogeográfico/Costa Pacífica. Bogotá: Universidad Nacional de Colombia; $997 \mathrm{pp}$

Rengifo F, Asprilla J, Jiménez A, Rengifo J, Castro A. 2002. Ecología y estructura de la comunidad de reptiles presentes en el corregimiento de Pacurita, municipio de Quibdó, Colombia. Quibdó: Corporación Nacional de Investigación y Fomento Forestal; 52 pp.

Rengifo J, Jiménez A, Asprilla J, Rengifo J, Roa Y, Moreno F. 2003. Distribución vertical y por sustrato de reptiles en un bosque pluvial tropical (bp-T) del Chocó. Revista Institucional Universidad Tecnológica del Chocó Diego Luis Córdoba. 18: 43-9.

Rengifo J, Asprilla J, Rengifo J, JiménezA, Roa Y. 2004. Una aproximación a la herpetofauna (anfibios y reptiles) del municipio de Novita, departamento del Chocó, Colombia. Revista Institucional Universidad Tecnológica del Chocó Diego Luis Córdoba. 20: 39-44.

Renjifo J, Lundberg M. 1999. Reptiles y anfibios de Urrá. Medellín: Editorial Colina. 96 pp.

Rentería L, Rengifo J. 2006. Revisión taxonómica del género Anolis (Squamata, Lacertilia: Polychrotidae) para el departamento del Chocó. En: Memorias XLI Congreso Nacional de Ciencias Biológicas, Quibdó

Rentería L, Rengifo J. Moya J. 2007. Comunidad de reptiles presente en el sotobosque de la selva pluvial central del departamento del Chocó. Revista Institucional Universidad Tecnológica del Chocó: Investigación, Biodiversidad y Desarrollo. 26 (2): 23-36.

Rentería L, Rivas T. 2009. Etnozoología y caracterización taxonómica de la ofidio-fauna asociada a cultivos agricolas presentes en la selva pluvial central del municipio de Quibdó, Chocó. En: Memorias del Primer Simposio de Herpetología en el Chocó Biogeográfico, Quibdó

Roa S, RuizCP. 1993. Anfibios contribución al proyecto estudio de la diversidad biótica de Colombia. Documento interno. Rangel O, Low P, Aguilar E (eds.). Bogotá: Convenio Inderena-Universida Nacional de Colombia.

Rueda-A JV, Carr JL, Mittermeier RA, Rodríguez-M JV, Mast RB, Vogt AGJ, et al. 2007. Las tortugas y los cocodrilianos de los países andinos del trópico. Serie Guías Tropicales de Campo $\mathrm{N}^{\circ}$ 6. Bogotá: Conservación Internacional; $538 \mathrm{pp}$.

Ruiz CP, Hernández J, Ardila M. 1993. La herpetofauna. En: Leyva P (ed.). Colombia Pacífico. Tomo 1. Bogotá: INDERENA, DND, GEF, PNUD; pp. 256-69.

Ruiz CP, Ardilla MR, Lynch J. 1996. Lista actualizada de la fauna amfibia de Colombia. Rev Acad Colomb. 20 (17): 65-415.

Vargas S, Bolaños M. 1999. Anfibios y reptiles presentes en hábitats perturbados de la selva lluviosa tropical en el Bajo Anchicaya, Pacífico colombiano. Rev Acad Colomb Cienc. 23 (suplemento especial): 499-511. 
Percepción, etimología y uso de la herpetofauna. LE Rentería Moreno et al.

Anexo 1

Herpetofauna registrada por la comunidad y los muestreos con su respectivo nombre nativo asignado por la comunidad indígena Embera-Dobida El Veintiuno

\begin{tabular}{|c|c|c|c|}
\hline Tipo de registro & Especies & Nombre común & Nombre Embera \\
\hline \multirow{17}{*}{$\begin{array}{l}\text { Especies registradas } \\
\text { en los muestreos }\end{array}$} & Rhaebo haematiticus & Sapo & Wililikiki, Bocorrózaque \\
\hline & Rhinella margaritifera & Spo & Wililikiki, Bocorrózaque \\
\hline & Craugastor fitzingeri & Rana & Wililikiki, Bocorrózaque \\
\hline & Craugastor longirostris & Rana & Wililikiki, Bocorrózaque \\
\hline & Oophaga histriónica & Rana Venenosa & Cuecue \\
\hline & Hypsiboas boans & Rana & Wililikiki \\
\hline & Scinax sugillatus & Rana & Wililikiki \\
\hline & Lithobates vaillanti & Rana & Wililikiki \\
\hline & Chironius carinatus & Cazadora negra & Oconto \\
\hline & Basiliscus galeritus & Chochora & Ochorró, Tolotolo, opoga \\
\hline & Gonatodes albogularis & Limpiacasa & Kekedama \\
\hline & Echinosaura horrida & Lagartija & Imicanasake \\
\hline & Anolis chloris & Izabandera & Oiga \\
\hline & Anolis granuliceps & Izabandera & Oiga \\
\hline & Anolis maculiventris & Izabandera & Oiga \\
\hline & Anolis peracce & Izabandera & Oiga \\
\hline & Anolis rivalis & Izabandera & Oiga \\
\hline \multirow{15}{*}{$\begin{array}{l}\text { Especies reportadas } \\
\text { por la comunidad }\end{array}$} & Caiman crocodylus & Babilla & Core \\
\hline & Kinosternom leucostomun & Tapa culo & Berreberre \\
\hline & Rhinoclemmys nasuta & Tortuga & Chibigui \\
\hline & Chelydra acutirostris & Bache & Pimporro \\
\hline & Bothrops asper & Equis & Equis \\
\hline & Lachesis muta & Berruguso & Birri \\
\hline & Porthidium nasutum & Equis 24 & lyara \\
\hline & Leptophis ahaetulla & Platanillo & Dama pa \\
\hline & Micrurus spp. & Coral & Corolachum \\
\hline & Boa constrictor & Jepa & Jempa \\
\hline & Bothrops punctata & Rabo de chucha & Nebe tama o Druchingachun \\
\hline & Diplogosus monotropis & Madre culebra & Colarina \\
\hline & Iguana iguana & Iguana verde & Opoga \\
\hline & Caecilia sp. & Ciega & Mevene \\
\hline & Bolitoglossa sp. & Salamandra & Moquitaparre \\
\hline
\end{tabular}

